\title{
Modelo Estrutural de Internalização e Externalização: Emergência, Validade e Utilidade Clínica
}

\author{
Hudson W. de Carvalho ${ }^{1}$ \\ Departamento de Psicologia da Universidade Federal de Pelotas, Pelotas, \\ Rio Grande do Sul, Brasil \\ Miguel Roberto Jorge \\ Departamento de Psiquiatria da Universidade Federal de São Paulo, São Paulo, \\ São Paulo, Brasil \\ Diogo Rizzato Lara \\ Faculdade de Biociências da Pontifícia Universidade Católica \\ do Rio Grande do Sul, Porto Alegre, Rio Grande do Sul, Brasil
}

\section{Resumo}

Os sistemas oficiais de classificação e diagnóstico em psicopatologia apresentam limitações relacionadas à extensa heterogeneidade clínica e ao significado dos padrões de comorbidade diagnóstica. A fim de superá-las, pesquisadores têm defendido uma estrutura dimensional para as psicopatologias mais prevalentes baseada em dois fatores latentes de internalização e externalização. O presente artigo visa avaliar a validade do Modelo Estrutural de Internalização e Externalização, tendo como norte a superação das referidas limitações e sua utilidade clínica. Revisão não sistemática da literatura nas bases Scielo e PubMed. Foram selecionados artigos empíricos e teóricos que avaliavam a pertinência do modelo. Foram excluídos estudos de replicação ou que avaliam o modelo em amostras infantis. A busca de literatura resultou em um total de 536 artigos, dos quais foram mantidos 44 . Esse conteúdo foi organizado em três linhas de pesquisa: estudos epidemiológicos-estruturais, psicobiológicos e psicométricos. O Modelo Estrutural de Internalização e Externalização apresenta indicadores que dão sustentabilidade aos seus pressupostos teóricos. O modelo é capaz explicar a comorbidade diagnóstica e, parcialmente, heterogeneidade clínica. Entretanto, não apresenta instrumental clínico normatizado para a operacionalização dos construtos e não há estudos com amostras clínicas graves.

Palavras-chave: Internalização, externalização, DSM, nosologia psiquiátrica, psicometria.

\section{Internalizing and Externalizing Structural Model: Emergency, Validity and Clinical Utility}

\begin{abstract}
The official systems of classification and diagnosis of psychopathology have limitations related to the extensive clinical heterogeneity and the meaning of patterns of diagnostic comorbidity. To overcome them, researchers have argued for a dimensional structure to the most prevalent psychopathology based
\end{abstract}

Endereço para correspondência: Departamento de Psicologia, Universidade Federal de Pelotas, Av. Duque de Caxias, 250, Fragata, Pelotas, RS, Brasil 96030-001. E-mail: hdsncarvalho@gmail.com Instituição onde o trabalho foi elaborado: Faculdade de Psicologia da Universidade Federal de Pelotas. Financiamento: Hudson W. de Carvalho foi bolsista de Pós-Doutorado Júnior (PDJ) do Conselho Nacional de Desenvolvimento Científico e Tecnológico (CNPq) durante a execução deste trabalho e Diogo R. Lara é bolsista de produtividade do CNPq nível 1B. 
on two latent factors of internalizing and externalizing vulnerability. The current study aimed to evaluate the validity of the Internalizing/Externalizing Structural Model, having as a standard its ability to overcome the aforementioned limitations and its clinical utility. A non-systematic review was performed in PubMed and SciELO databases. Theoretical and empirical articles were retrieved based and evaluated on basis of its relevance to the model. Replications and studies based on samples of children were excluded. Bibliographic search yielded a total of 536 articles, of which 44 were retrieved. The content of the articles were organized into three lines of research: structural-epidemiological, psychobiological, and psychometric. The Internalizing/Externalizing Structural Model presents indicators that support its theoretical assumptions. The model is able to explain the diagnostic comorbidity and, partially, clinical heterogeneity. However, there is no standardized clinical instrument for the operationalization of internalizing/externalizing constructs and no validation studies with inpatient samples.

Keywords: Internalizing, externalizing, DSM, psychiatric nosology, psychometrics.

\section{El Modelo Estructural de Internalización y Externalización: Emergencia, Validez y Utilidad Clínica}

\section{Resumen}

Los sistemas oficiales de clasificación y diagnóstico de la psicopatología tienen limitaciones relacionadas con la amplia heterogeneidad clínica y el significado de los patrones de comorbilidad diagnóstica. Para superarlas, los investigadores han argumentado a favor de una estructura tridimensional de la psicopatología más frecuente en base a dos factores latentes de internalización y externalización vulnerabilidad. El presente estudio tuvo como objetivo evaluar la validez del modelo estructural internalización/ externalización tener patrones de utilidad, la heterogeneidad y la comorbilidad clínicas como parámetros. Una revisión no sistemática fue realizada en bases de datos PubMed y SciELO. Artículos teóricos y empíricos se recuperaron basa y evaluado en función de su relevancia para el modelo. Se excluyeron réplicas y estudios basados en muestras de niños. Búsqueda bibliográfica arrojó un total de 536 artículos, de los cuales 44 fueron recuperados. El contenido de los artículos que se han organizado en tres líneas de investigación: estructural-epidemiológicos, psicobiológicos y psicométricos. El modelo estructural de internalización/externalización presenta indicadores que apoyan sus hipótesis teóricas. El modelo es capaz de explicar la comorbilidad de diagnóstico y, en parte, la heterogeneidad clínica. Sin embargo, no existe un instrumento clínico estandarizado para avaluación de internalización e externalización, ni estudios de validación con muestras de pacientes hospitalizados.

Palabras clave: Internalización, externalización, DSM, la nosología psiquiátrica, psicometría.

Os sistemas contemporâneos de classificação psiquiátrica (American Psychiatric Association [APA], 2002; Organização Mundial de Saúde [OMS], 1991) organizam os transtornos mentais a partir de categorias nosológicas (p.ex., transtorno depressivo maior, transtorno de pânico e transtornos relacionados ao uso de substâncias). Tais categorias, por sua vez, são estruturadas em classes diagnósticas (i.e., transtornos de humor, transtornos de ansiedade, transtornos relacionados ao uso de substâncias, entre outros) e eixos temáticos (i.e., eixo I, transtornos clínicos; eixo II, transtornos da personalidade e retardo mental). Nesses sistemas, os indivíduos são classificados como membros ou não membros de uma determinada categoria diagnóstica, sem qualquer possibilidade de gradação (APA, 2002; OMS, 1991).

O referido entendimento sobre psicopatologia tem encontrado pouco suporte empírico e imposto limitações importantes tanto para clínicos quanto para pesquisadores, particularmen- 
te no que tange ao significado da comorbidade psiquiátrica e à hetegeneidade clínica (Clark, Watson, \& Reynalds, 1995; Krueger \& Markon, 2006a, 2006b; Maj, 2005; Watson, 2005; Widiger \& Clark, 2000; Widiger \& Sankis, 2000). A fim de superá-las, diferentes grupos de pesquisa têm defendido uma estrutura dimensional para a psicopatologia, na qual dois fatores latentes moderadamente correlacionados $(r \cong 0,5)$ - internalização e externalização - explicam a variância comum entre os transtornos mentais mais prevalentes (de Carvalho, Andreoli, Vaidyanathan, et al., 2013; Krueger, 1999; Krueger, Caspi, Moffit, \& Silva, 1998; Slade \& Watson, 2006; Vollebergh et al., 2001). Internalização é um fator que explica a covariância entre transtornos relacionados ao humor depressivo unipolar, à ansiedade e ao medo. Essa dimensão é, por vezes, subdividida em dois fatores correlacionados $(r \cong 0,7)$ de ansiedade-sofrimento ou de angústia (conformado a partir dos transtornos depressivos unipolares, transtorno de ansiedade generalizada e transtorno de estresse pós-traumático) e medo (conformado a partir dos transtornos obsessivo-compulsivo, fóbicos e de pânico). Externalização é um fator que explica a covariância entre transtornos relacionados ao comportamento disruptivo e antissocial e ao uso nocivo de substâncias.

O principal argumento dos proponentes do Modelo Estrutural de Internalização e Externalização em seu favor reside na construção de um sistema empiricamente sustentada e teoricamente consistente para a classificação e o diagnóstico psiquiátrico (Krueger \& Markon, 2006a, 2006b). Desse modo, o modelo ofereceria um "mapa" conceitual para se entender os padrões de comorbidade e a heterogeneidade clínica (Krueger \& Markon, 2006a).

O presente artigo apresenta três objetivos interligados. Inicia-se com uma breve descrição do modelo categorial de classificação e diagnóstico em psicopatologia, ressaltando as limitações que motivaram o desenvolvimento do Modelo Estrutural de Internalização e Externalização. Segue-se com uma revisão ampla e integrativa, porém não sistemática, dos principais trabalhos sobre a emergência e o desenvolvimento do mo- delo em questão. Por fim, realiza-se uma apreciação da pertinência do Modelo Espectral de Internalização e Externalização como paradigma classificatório e diagnóstico dos transtornos mentais, tendo como norte a sua capacidade em superar os desafios impostos pela comorbidade diagnóstica e a heterogeneidade clínica e, sobretudo, o conceito de utilidade clínica (First, 2005; Reed et al., 2013).

\section{Método}

O presente estudo é uma revisão não sistemática da literatura relacionada ao Modelo Estrutural de Internalização e Externalização. Múltiplas estratégias de busca bibliográfica foram utilizadas a fim de identificar artigos, capítulos de livro, teses e dissertações que versassem sobre o referido modelo e temas relacionados, como epidemiologia psiquiátrica, comorbidade, utilidade clínica de diagnósticos e relação entre temperamento/personalidade e psicopatologia. Utilizou-se, sobretudo, os sítios da PubMed e SciELO. Os critérios de busca abrangeram idioma (inglês e português), período de publicação demarcado pela proposição original do modelo (janeiro de 1998) até fevereiro de 2013 e estudos com amostras de adolescentes e adultos somente.

O levantamento de dados valeu-se de diferentes indexadores de conteúdo (palavras-chave e título/resumo) para a seleção de estudos originais e teóricos, a saber: externalização (externalizing), internalização (internalizing) e estrutura dos transtornos mentais (structure of mental disorders). Apenas artigos que representasse, à sua época, ineditismo e avanço empírico, metodológico ou conceitual foram incluídos, isto é: estudos de replicação do modelo que não acrescentasse novidades como, por exemplo, ampliação no número de variáveis ou amostras não estudadas, foram excluídos devido ao seu papel coadjuvante no desenvolvimento e formalização do modelo estrutural de internalização e externalização.

Outras produções bibliográficas (capítulos de livro e ensaios críticos) foram incluídas por terem sido identificadas nos artigos de referência 
como parte do racional teórico relacionado ao Modelo Estrutural de Internalização e Externalização. O critério de escolha se deu com base na repetição com que esses artigos eram citados nos estudos empíricos sobre o modelo.

\section{Resultados}

Foram identificados 536 artigos no PubMed e 11 no Scielo. Após a leitura dos resumos foram excluídos trabalhos que não abordavam o Modelo Estrutural de Internalização e Externalização e estudos de replicação. Esse procedimento resultou em um total de 23 artigos que apresentaram evidências que corroboraram o modelo e 16 artigos teóricos e de revisão que avaliavam sua pertinência como modelo nosológico.

Os estudos identificados foram organizados em três linhas gerais: (a) análises estruturais de dados epidemiológicos; (b) estudos cuja meta era estabelecer a base hereditária (genética comportamental) e neurobiológica da internalização e externalização; (c) estudos de orientação psicométrica. Essa organização refletiu as principais tendências de pequisa sobre o Modelo Estrutural de Internalização e Externalização. As Tabelas 1 e 2 do Apêndice destacam os artigos incluídos no presente estudo.

\section{Do Diagnóstico Psiquiátrico e suas Limitações}

Os sistemas oficiais de classificação e diagnóstico dos transtornos mentais, atualmente representados pelo DSM-IV (APA, 2002) e pela décima edição da Classificação Internacional de Doenças (CID-10; OMS, 1991), são pretensamente ateóricos e predominantemente descritivos. A organização interna desses sistemas responde principalmente a princípios de conveniência semântica e de utilidade clínica (First, 2005). O DSM-V (APA, 2013) pretende superar esse caráter explicitando seu viés patofisiológico, tendo a organização interna do manual sido modificada a fim de agrupar transtornos que compartilhassem de determinantes neurobiológicos. A crítica apresentada se mantem atual, uma vez que o procedimento diagnóstico continua semelhante (eminentemente clínico e fenomenológico) e baseado em um modelo de classificação por meio de categorias. A análise que se segue é centrada nos sistemas DSM-IV e CID-10, uma vez que o Modelo Estrutural de Interna e Externalização foi construído com base em variáveis providas por esses sistemas e motivadas pelas críticas que lhes foram endereçadas.

O DSM-IV e a CID-10 concebem os transtornos mentais como variáveis discretas e independentes. Esse entendimento sobre psicopatologia tem encontrado pouco suporte e imposto uma série de limitações, a saber:

1. Visão fragmentada da atividade mental devidoa concepçãocategorial eindependente dos transtornos mentais - contrastante com evidências de que domínios psicológicos e estruturas neurobiológicas funcionam de modo integrado e coordenado (Lara et al., 2012; Meehl, 1992; Ofrat \& Krueger, 2012);

2. Inflação de categorias nosológicas e de comorbidades (Clark et al., 1995; Maj, 2005);

3. Excessiva heterogeneidade clínica (Krueger \& Markon, 2006a; Watson, 2005; Widiger $\&$ Sankis, 2000);

4. Incapacidade de explicar o porquê de uma mesma classe de medicação ser eficaz em diferentes classes de transtorno mental (Lara \& Souza, 2001);

5. Ausência de parâmetros claros que caracterizem saúde mental (Lara et al., 2012);

6. Dificuldade para se relacionar com áreas afins do conhecimento, como a psicologia e as neurociências (Insel \& Quirion, 2005).

Outra importante característica dos critérios de diagnósticos para transtornos mentais é refere-se ao seu foco. Este se restringe ao estado de funcionamento atual (o que a pessoa tem estado) e despreza o modo cotidiano da pessoa de sentir, pensar e agir (como a pessoa é - traço). Ignora-se, desse modo, o temperamento (Clark, 2005; Lara et al., 2012) do indivíduo no entendimento de sua condição atual de funcionamento mental e socioafetivo. Esse posicionamento é contrastante com o conjunto crescente de evidências que mostra que os traços do temperamento e da personalidade são essenciais ao 
entendimento da incidência, do prognóstico, da comorbidade e da efetividade de tratamentos (Clark, 2005; Cloninger, Svrakic, \& Przybeck, 1993; de Carvalho, Andreoli, Lara, et al., 2013). Além disso, a maioria dos transtornos psiquiátricos apresenta um padrão recorrente e crônico, aproximando-se mais da noção de traço (i.e. do modo de ser das pessoas no cotidiano) do que de estado (Möller, 2008).

No DSM-IV (APA, 2002), os transtornos da personalidade são localizados no Eixo II, enquanto os transtornos clínicos são posicionados no Eixo I. O Eixo II ressalta a importância de considerar o modo cotidiano de ser do paciente (seu temperamento ou personalidade). Todavia, não é apresentado um racional teórico e operacional claro para o construto personalidade e não há delimitação de parâmetros de normalidade ou saúde da personalidade. No DSM-V (APA, 2013), apesar de realocar os Transtorno da Personalidade e reconhecer a importância da avaliação de traços da personalidade, o mesmo não se sucede: não uma proposta clara sobre qual racional teórico deveria ser adotado e, consequentemente, qual instrumento deveria ser adotado. Assim, o conjunto de avanços da psicologia sobre o entendimento e avaliação do temperamento, da personalidade e de diferenças individuais continua sendo desconsiderado no diagnóstico nosológico.

O conjunto das referidas limitações configurou um contexto no qual modelos alternativos de diagnóstico e de classificação dos transtornos mentais têm sido propostos. Dentre os quais, o Modelo Estrutural de Internalização e Externalização tem relativo destaque devido a sua influência nas propostas de reformulação dos sistemas DSM e CID, particularmente no que tange à heterogeneidade clínica, ao significado dos padrões de comorbidade e na explicação da relação entre psicopatologia e traços das diferenças individuais.

\section{Emergência do Modelo Espectral de Internalização e Externalização}

O modelo estrutural de internalização e externalização surgiu a partir da avaliação da estru- tura latente de um número limitado de transtornos mentais relacionados ao humor depressivo unipolar, à ansiedade, ao medo, ao uso de substâncias e à conduta antissocial. Esses primeiros estudos foram realizados com base em dados provenientes de pesquisas epidemiológicas de base comunitária (Krueger, 1999; Krueger et al., 1998; Vollebergh et al., 2001). Esse desenho se mostrou bem sucedido, influenciando pesquisas mais recentes que ampliaram o escopo original tanto pela inclusão de um número mais amplo de psicopatologias (Kessler et al., 2011; Røysamb et al., 2011) quanto pelo exame da estrutura fatorial de trantornos mentais em amostras não comunitárias, como a de prisioneiros em regime fechado (de Carvalho, Andreoli, Vaidyanathan, et al., 2013). Uma segunda abordagem objetivou estabelecer as bases genéticas (Kendler, Prescott, Myers, \& Neale, 2003; Krueger et al., 2002) e marcadores biológicos dos construtos internalização e externalização (Ofrat \& Krueger, 2012; Patrick, 2008; Vaidyanathan, Nelson, \& Patrick, 2012). A terceira linha de pesquisa serviu-se da psicometria moderna para avaliar a validade dos construtos internalização e externalização e identificar os elementos emocionais, sociais e comportamentais constitutivos dos referidos construtos (de Carvalho, Pinheiro, Patrick, Krueger, \& Markon, 2007; Krueger, Markon, Patrick, Benning, \& Kramer, 2007).

\section{Estudos de Orientação Epidemiológico- -Estrutural}

No primeiro estudo publicado, Krueger et al. (1998) avaliaram a estrutura latente de dez transtornos mentais (transtorno depressivo maior [TDM]; distimia [DIS]; transtorno de ansiedade generalizada [TAG]; agorafobia [AGF]; fobia social $[\mathrm{FS}]$ fobia específica $[\mathrm{FE}]$; transtorno obsessivo-compulsivo [TOC]; transtorno de conduta [TC]; transtorno de dependência de cannabis e transtorno de dependência de álcool) e a estabilidade dessa estrutura avaliando uma amostra comunitária ampla e representativa de uma cidade neozelandesa (Dunedin) aos 18 de depois ao 21 anos de idade. Neste estudo, utilizaram-se diagnósticos baseados nos critérios referentes ao DSM-III-R. 
Os resultados, baseados em modelagem estrutural, indicaram que um modelo de dois fatores correlacionados de internalização e externalização era o que melhor representava as correlações entre as síndromes avaliadas. Os TDM, DIS, TAG, TOC, AGF, FS e FE apresentaram substanciais cargas fatoriais no fator de internalização, enquanto TC e dependência de substâncias o fizeram no fator de externalização. Ambos fatores apresentaram elevada estabilidade temporal, tendo o fator de externalização mostrado sutil, porém maior estabilidade que o fator de internalização. Krueger et al. (1998) acabaram por definir o significado psicológico de internalização e externalização a partir de disposições individuais em relação ao mundo: internalização seria uma tendência de escape socioafetivo por meio da retração social, do medo ou de pensamentos autorreferentes. Externalização, por sua vez, foi definida como uma orientação geral que coloca o indivíduo contra o mundo, seja pela criminalidade, seja pelo desrespeito às regras.

Em um estudo subsequente, Krueger (1999) examinou a estrutura de dez síndromes psiquiátricas em uma amostra comunitária, representativa e probabilística de norte-americanos adultos $(N=8,098)$. Os dados foram examinados por meio de análises fatoriais confirmatórias para a amostra total, separadamente para homens e mulheres, e para uma subdivisão da amostra que buscava auxílio relativo à saúde mental paralelamente ao momento em que a coleta de dados ocorreu. Quatro modelos fatoriais foram propostos (um modelo unifatorial, um modelo de dois fatores, um modelo com três e um com quatro fatores) e, então, comparados em termos de seu ajuste (geral, incremental e parcimonioso) aos dados. O modelo de três fatores foi o que apresentou melhor ajuste parcimonioso, tendo sido selecionado como o mais adequado. Os diagnósticos de dependência de álcool, dependência de outras drogas e transtorno de personalidade antissocial (TPA) foram explicados pelo primeiro fator (externalização), TDM, DIS e TAG pelo segundo fator (ansiedade-sofrimento) e FE, AGF e transtorno de pânico (TP) pelo terceiro fator (medo). A elevada correlação entre os fatores de ansiedade-sofrimento e de medo $(0,73)$ sugeriram que estes seriam mais apropriadamente compreendidos como indicadores latentes (subfatores) de um fator de ordem-superior (fator de internalização). Os fatores de internalização e externalização apresentaram correlação positiva e moderada $(0,51)$.

Vollebergh et al. (2001) replicaram os achados de Krueger (1999) e Krueger et al. (1998) em uma amostra representativa e aleatória da população holandesa. Nesse estudo, a estrutura e a estabilidade (um ano entre a primeira e segunda coleta de dados) de nove transtornos mentais (TDM, DIS, TAG, FS, FE, AGF, TP, dependência de álcool e dependência de drogas) foram analisadas por meio de equações estruturais em um delineamento epidemiológico longitudinal. Os resultados replicaram a estrutura encontrada por Krueger (1999) e, além disso, os fatores de internalização e de externalização mostraram alta estabilidade pelo período de um ano.

Com base em dados provenientes de 14 países, dentre os quais o Brasil se incluía, Krueger, Chentsova-Dutton, Markon, Goldberg, e Ormel (2003) avaliaram a estrutura latente de sete indicadores de transtorno mental, a saber: uso nocivo de álcool, depressão, somatização, hipocondria, neurastenia, preocupação e excitabilidade ansiosa. Os resultados indicaram que uma estrutura bifatorial se ajustava bem aos dados, na qual uma dimensão consistente de internalização saturava diretamente a variância comum dos indicadores relacionados à depressão e ansiedade (fator de internalização) e outra correlacionada ao uso nocivo de álcool.

Krueger et al. (2002) testaram se a comorbidade entre síndromes externalizantes seria mais adequadamente descrita como indicadora de uma dimensão contínua (Modelo de traço latente) ou como categorias diagnósticas ordenadas por gravidade (Modelo de classe latente). Os resultados indicaram que o Modelo unifatorial de traço latente representa com maior verossimilhança os transtornos externalizantes e que a dimensão extraída apresenta distribuição normal e é invariante em relação ao sexo.

Slade e Watson (2006) corroboraram os achados de Krueger (1999) ao avaliar a estrutura latente de 10 e 11 transtornos mentais comuns 
com base nos sistemas DSM-IV e CID-10, respectivamente. Os resultados apontaram para um modelo contendo três fatores latentes: externalização, medo e ansiedade-sofrimento. Os dois últimos (medo e ansiedade-sofrimento) foram redesenhados como indicadores latentes de um fator de ordem-superior (internalização). Esse estudo foi o primeiro a usar os critérios do CID10 e a mostrar a convergência de resultados estruturais entre os sistemas da OMS e da APA. Ademais, apresenta a virtude de utilizar uma amostra ampla e representativa da população australiana.

Em uma meta-análise, Krueger e Markon (2006a) mostraram a pertinência do modelo de internalização (com subfatores de ansiedade/sofrimento e medo) e externalização com base em cinco estudos previamente publicados. Esse estudo mostrou a robustez do modelo, replicando-o conforme o delineado por Krueger (1999).

A estrutura do fator de internalização foi então revisitada por Seeley, Kosty, Farmer, e Lewinsohn (2011) que fizeram uso de diagnósticos depressivos unipolares (TDM e DIS) e de ansiedade (TAG, TOC, Transtorno do Estresse Pós-Traumático [TEPT], transtornos de ansiedade de separação [FE, FS e TP]) em uma amostra ampla de adultos. Três estruturas foram testadas: um modelo unidimensional, um modelo baseado na estrutura proposta pelo DSM-IV (um fator para transtornos depressivos unipolares e outro para transtornos de ansiedade) e um modelo estruturado conforme a proposta de Krueger (1999). Os resultados se mostraram inconclusivos, pois não houve diferença significativa em relação à qualidade do ajuste dos modelos delineados: todas as estruturas apresentaram excelente ajuste aos dados.

O trabalho de Seeley et al. (2011) é de particular relevância, pois os resultados indicam que não há diferença significativa - com base em indicadores de ajuste de modelo (goodness of fit) produzidos a partir da modelagem por equação estrutural - entre o modo como o DSM-IV organiza os transtornos mentais e a proposta alternativa de Krueger (1999) para os transtornos depressivos e de ansiedade. Estes autores concluem ser prematuro modificar o DSM com base na proposta de uma classe de transtornos fóbicos (fator de medo) e de sofrimento (fator de ansiedade-sofrimento).

Outros estudos recentes têm colocado dúvidas sobre a forma mais adequada de estruturar o fator de internalização. Kessler et al. (2011) ao examinarem a função dos fatores de internalização e externalização no desenvolvimento de comorbidades ao longo da vida, sugerem uma estrutura menos diferenciada (fator-simples), na qual o fator de internalização não é subdividido em dois fatores de ansiedade-sofrimento e medo. Røysamb et al. (2011) evidenciam a consistência das dimensões de internalização e externalização, mesmo ao avaliarem um número mais amplo de transtornos mentais, porém, como dimensões de primeira-ordem (modelo de fator-simples).

Forbush e Watson (2013) analisaram a estrutura latente de um espectro amplo de transtornos mentais (transtornos de humor uni e bipolar, transtornos de ansiedade, transtornos alimentares, transtornos da personalidade e relacionados ao uso de substâncias) e, baseados em diversos índices de ajuste de modelo, encontraram fortes evidências de validade estrutural para um modelo de dois fatores baseado nas dimensões de internalização e externalização. O último continuou a ser delineado como um fator simples, enquanto internalização apresentou múltiplas facetas, a saber: angústia, medo, problemas alimentares, disforia e bipolaridade.

$\mathrm{O}$ único estudo até então publicado que examinou a estrutura de transtornos mentais em uma amostra de prisioneiros em regime fechado mostrou suporte a um modelo no qual internalização e externalização são definidos como dimensões simples (de Carvalho, Patrick, Jorge, \& Andreoli, 2011). Os autores hipotetizaram que a não divisão de internalização em dois subfatores poderia ser explicada pela excessiva morbidade psiquiátrica no contexto prisional: como a prevalência e comorbidade são muito altas, pode ser difícil diferenciar empiricamente ansiedade/ sofrimento e medo. Todavia, esse estudo replica a estrutura básica proposta por Krueger (1999) em uma amostra nunca antes estudada que dá suporte adicional à robustez do modelo. 
Os estudos ora apresentados foram baseados em dados diagnósticos dicotômicos (presença ou ausência do transtorno). Visando superar essa limitação, Wright et al. (2013) examinaram a estrutura latente de indicadores quantitativos (quantidade de sintomas) de um espectro amplo de psicopatologias. Os resultados indicaram que externalização se mantem como um fator único, porém internalização foi subdividida em subfatores de angústia, medo e um terceiro que explicou os sinais e sintomas relacionados ao TOC que, por sua vez, mostrou menor grau de saturação no fator de internalização. O estudo de Wright et al. (2013) trouxe evidências de que as psicoses pertencem a um fator independente, porém mais proximamente relacionado à internalização que à externalização. A maior proximidade com internalização se deve, provavelmente, ao fato de que os sintomas negativos (que apresentam caráter internalizante) são mais persistentes e pervasivos que os sintomas positivos (que apresentam caráter mais externalizantes, como a paranoia). Por fim, o estudo mostrou por meio de comparação de modelos (categorial $v s$ misto $v s$ dimensional) que a psicopatologia é um fenômeno dimensional, isto é, contínuo.

No Brasil, um estudo recente (de Carvalho et al., 2014), contando com uma amostra de 3.728 adultos, mostrou que a covariância entre o traço Afeto Negativo (AN; predisposição a vivenciar o cotidiano com raiva, medo, angustia e ansiedade), transtorno depressivo maior e transtornos de ansiedade podem ser explicadas por dois fatores correlacionados de ansiedade-sofrimento e medo. Além de replicar a estrutura proposta para fator de internalização, este estudo mostra que o traço AN pode ser delimitado como uma faceta do fator de ansiedade-sofrimento. $\mathrm{O}$ autor propôs uma interpretação clínica para as facetas da internalização: o fator de medo parece estar associado a condutas de evitação de ambientes externos, onde o indivíduo pode se sentir a mercê do imprevisto (como um possível ataque de pânico), enquanto o fator de ansiedade-sofrimento parece não apresentar essa relação.

O conjunto das evidências referentes a estudos de orientação epidemiológico-estrutural mostra que o Modelo Estrutural de Internalização e Externalização é empiricamente pertinente.
Entretanto, a definição de internalização como unidimensional ou como um fator de ordem-superior requer, claramente, estudos adicionais. Ressalta-se, entretanto, que o os estudos avançaram de modelo mais restritos e baseados unicamente em diagnósticos (Krueger, 1999; Slade \& Watson, 2006), para modelos mais amplos e que utilizam variáveis diagnósticas e traços do temperamento (de Carvalho et al., 2014).

\section{Estudos de Orientação Psicobiológica}

Tradicionalmente, pesquisas sobre genética e biomarcadores de psicopatologias focam apenas um transtorno de interesse, como, por exemplo, TDM ou TEPT. Esse método falha em discriminar entre dimensões gerais, que explicam a coocorrência entre transtornos, e específicas, que permitem a diferenciação de transtornos associados.

Sob o contexto do Modelo Estrutural de Internalização e Externalização, estudos de genética comportamental (Kendler et al., 2003; Krueger et al., 2002) e sobre biomarcadores objetivam clarificar as fontes da homogeneidade de grupos de transtornos (Hall, Bernat, \& Patrick, 2007; Patrick et al., 2006). Configuram-se as expectativas de que o avanço do conhecimento venha fomentar uma classificação psiquiátrica empírica e teoricamente orientada e de que o diagnóstico também se estabeleça com base em biomarcadores confiáveis.

No primeiro estudo publicado, Krueger et al.(2002) avaliaram a estrutura das contribuições genéticas e ambientais para o desenvolvimento do fator de externalização em uma amostra de 626 pares de gêmeos (411 pares monozigóticos e 215 pares dizigóticos) adolescentes e de ambos os sexos, com relação aos diagnósticos de TC, TPA, dependência de álcool, dependência de outras drogas e traço desinibição comportamental/ impulsividade (unconstrained, traço avaliado pela aplicação do Multidimensional Personality Inventory por Tellegen, 1982). Os resultados indicaram excelente ajuste para um modelo unifatorial, no qual um fator (externalização) saturou diretamente a variância comum das variáveis empíricas (modelo de caminho comum). Ainda, o modelo delineado se mostrou invariante em relação ao sexo dos respondentes, sendo $81 \%$ das diferenças individuais no nível do traço latente 
explicadas por variância genética aditiva (hereditariedade simples). Observaram-se, sobre todas as variáveis examinadas, um efeito significativo do ambiente compartilhado sobre a manifestação do TC, um efeito genético específico sobre a manifestação do traço de personalidade incontida e um efeito do ambiente não compartilhado. Outro fato relevante ao desenvolvimento do Modelo Estrutural de Externalização se deveu à inclusão de uma medida psicométrica de personalidade junto a diagnósticos psiquiátricos como indicador latente do fator de externalização.

Kendler et al. (2003) replicaram o modelo de internalização e externalização em uma amostra de 5.600 gêmeos monozigóticos e dizigóticos de ambos os sexos, com médias de idade significativamente superiores às do estudo de Krueger et al. (2002). Eles identificaram dois fatores genéticos (hereditariedade simples, aditiva) invariantes em relação ao sexo que explicavam diretamente $83 \%$ da covariância de sete síndromes: TDM, TAG, fobia, dependência de álcool, dependência/abuso de drogas, TPA e TC. O fator de internalização foi subdividido, conforme proposto por Krueger (1999) e Vollebergh et al. (2001), em dois subfatores de ansiedade-sofrimento e medo.

Hettema, Neale, Myers, Prescott e Kendler (2006) examinaram como fatores genéticos e ambientais comuns ao neuroticismo e sete transtornos internalizantes poderiam explicar padrões de comorbidade. A amostra contou com 9.000 gêmeos uni e bivitelinos. Os resultados indicaram que há um compartilhamento substancial, porém não completo, de fatores genéticos que influenciam variações individuais em neuroticismo e transtornos internalizantes. Um fator genético comum de risco entre neuroticismo e transtornos internalizantes explicou aproximadamente um terço do risco para o desenvolvimento de psicopatologias, mas fatores genéticos específicos na determinação do neuroticismo explicaram parte da suscetibilidade à psicopatologia internalizante. Com base nesses achados, os autores sugerem que neuroticismo seja uma variável fundamental para o entendimento de padrões de comorbidade entre transtornos depressivos e fóbico-ansiosos.

Patrick et al., (2006) em um estudo de orientação psicofisiológica, avaliaram a relação entre o P300 e a dimensão de externalização. P300 é um potencial evocado positivo que ocorre 300 milisegundos após a apresentação de um estímulo e está relacionado à avaliação ou categorização cognitiva de um estímulo, assim como na alocação da atenção e no processamento de estímulos novos. Pesquisas identificaram que indivíduos com dependência de substâncias e conduta antissocial apresentam menores amplitudes de P300. Assim, Patrick et al. (2006) propuseram um estudo que pretendiam verificar se (a) a amplitude do P300 e o fator de externalização estariam associados, (b) se as conexões entre externalização e $\mathrm{P} 300$ poderiam explicar as relações entre dependência de álcool e P300, e (c) se a amplitude do P300 poderia ser considerada um indicador da dimensão de externalização, em vez de um indicador específico de vulnerabilidade ao uso de substâncias. Os resultados mostraram uma clara associação entre externalização e menores amplitudes do P300 na amostra estudada (969 adolescentes do sexo masculino). A relação entre os diagnósticos específicos avaliados (dependência de álcool, drogas e nicotina, TC e TPA) e a amplitude do P300 foi explicada pela variância comum entre as síndromes avaliadas (fator de externalização) e não pela variância específica de cada uma delas. Além disso, ao se computarem os diagnósticos e a amplitude do P300 em uma matriz e rodar uma análise de componentes principais, observou-se que apenas um componente era capaz e suficiente para explicar a maior parte da variância compartilhada. Por conseguinte, conjecturou-se que a amplitude do P300 seja um endofenótipo do fator de externalização.

Outro estudo psicofisiológico avaliou se escores mais elevados em uma medida ampla da externalização poderiam predizer amplitudes reduzidas de negatividade relacionada ao erro, um potencial evocado relacionado ao automonitoramento e que se encontra reduzido em síndromes externalizantes (Hall et al., 2007). Negatividade relacionada ao erro é observada 100 milisegundos (N100) após o indivíduo sob exame cometer erros em uma tarefa de multipla escolha, mesmo quando este não está consciente do erro. Os resultados desta pesquisa corroboraram a hipótese original do estudo, mostrando amplitudes 
menores de negatividade relacionada ao erro em indivíduos mais externalizantes. Os achados ainda sugerem que déficits neurobiológicos na capacidade de automonitoramento podem ser um importante fator endógeno de vulnerabilidade ao desenvolvimento de um amplo espectro de problemas relacionados ao controle de impulsos.

Por meio de uma ampla revisão de literatura baseada nos substratos cerebrais relacionados à reatividade emocional e regulação afetiva, $\mathrm{Pa}$ trick e Bernat (2006) delinearam o fundamento neurobiológico da internalização e da externalização. Dois complexos subcorticais denominados de sistema motivacional defensivo e sistema motivacional apetitivo foram identificados como as bases emocionais da internalização e da externalização, respectivamente. O núcleo central da amigdala - responsável pela ativação do medo - e o núcleo estriado ventral - relacionado à ativação da ansiedade - foram estabelecidos como base do sistema motivacional defensivo. Essas estruturas são centrais à aprendizagem do medo e da ansiedade e diferenças individuais quanto à reatividade prediriam o grau de vulnerabilidade internalizante. A via dopaminérgica mesolímbica, responsável pela conduta apetitiva e pela suscetibilidade ao aprendizado por reforço positivo, seria a base neurobiológica comum à vulnerabilidade externalizante. Há evidências adicionais de que os receptores D4 de Dopamina mediam a relação entre tendência à externalização e inteligência geral e, logo, funções executivas (DeYoung et al., 2006).

A regulação desses vetores emocionais se daria com base em funções cognitivas superiores relacionadas à representação de metas e por meio de um processo ora inibitório, ora excitatório, do tipo top down. Quatro estruturas corticais foram identificadas: as porções dorsolateral e orbitofrontal do córtex pré-frontal, o hipocampo e o córtex cingulado anterior. De modo geral, o funcionamento conjunto dessas estruturas possibilitaria ao indivíduo representar metas, automonitorar-se e inibir respostas prepotentes, o que impediria manifestações emocionais incompatíveis com a execução das metas mentais.

Com base no modelo descrito por Patrick e Bernat (2006), pode-se definir internalização e externalização como dimensões emocionais básicas de funcionamento independente que se mostram relacionadas através de processos superiores de monitoramento e regulação. A Figura 1 traz uma representação do modelo psicobiológico de internalização e externalização.

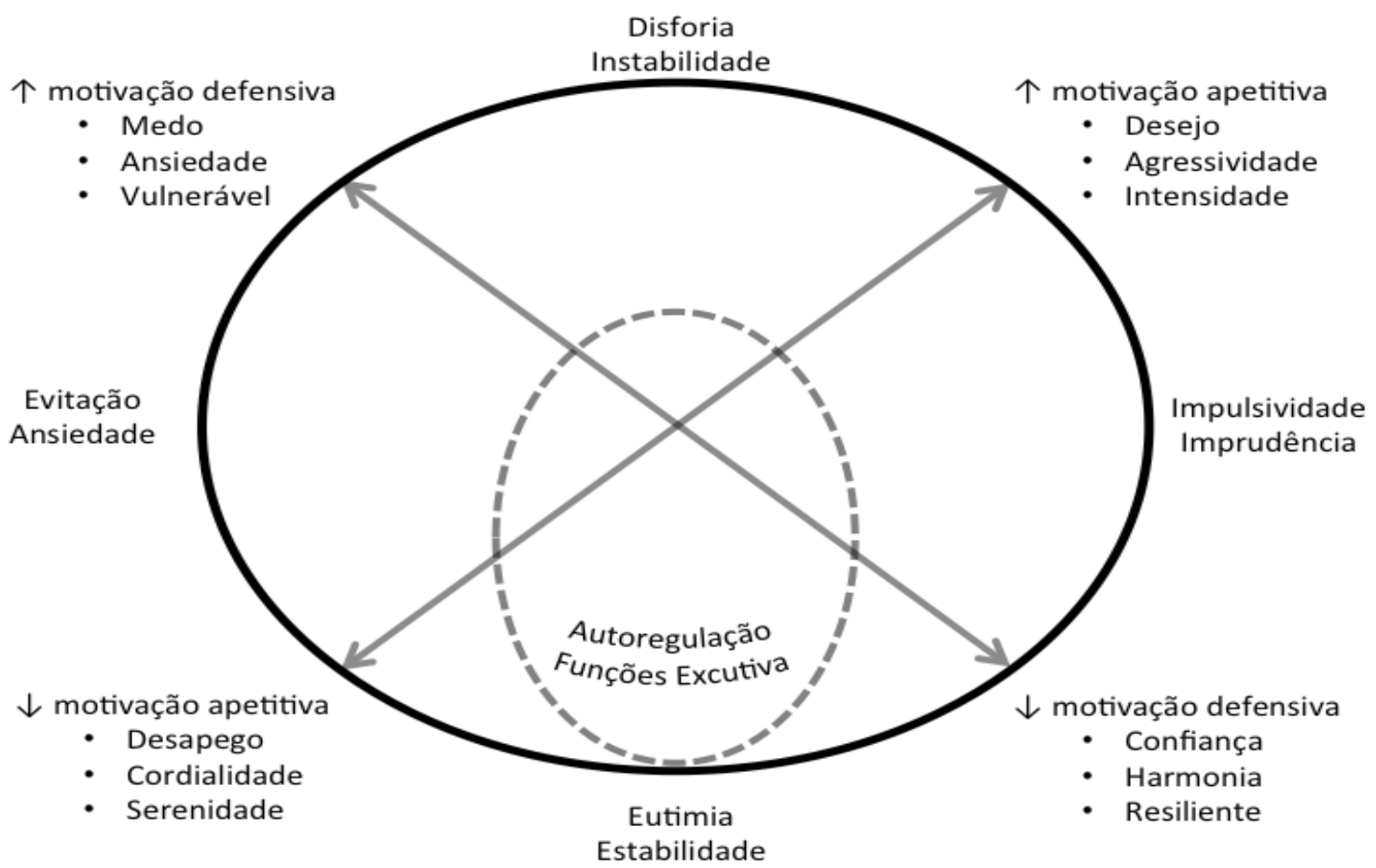

Figura 1. Modelo psicobiológico de internalização e externalização baseado em Patrick e Bernat, 2006. 


\section{Estudos de Orientação Psicométrica}

Krueger et al. (2002) testaram se a comorbidade entre síndromes externalizantes seria mais adequadamente descrita como indicadora de uma dimensão contínua (Modelo de traço latente) ou como categorias diagnósticas ordenadas por gravidade (Modelo de classe latente). Os resultados indicaram que o Modelo unifatorial de traço latente representa com maior verossimilhança os transtornos externalizantes e que a dimensão extraída apresenta distribuição normal e é invariante em relação ao sexo.

Partindo de um modelo logístico de dois parâmetros da Teoria de Reposta ao Item, McGlinchey e Zimmerman (2007) examinaram a estrutura de cinco transtornos internalizantes (TDM, TAG, pânico com agorafobia, FE e FS) em uma ampla amostra de pacientes ambulatoriais (2.300 participantes). Os resultados indicaram que um fator explicou diretamente a covariância entre os cinco indicadores psiquiátricos utilizados, que os transtornos se localizam na porção superior da distribuição desse traço latente e que internalização se mostra associada a três indicadores de sofrimento psicossocial: funcionamento social insuficiente, tempo de afastamento do trabalho e tempo de hospitalização.

Outro estudo (Krueger et al., 2007) delineou o espectro da externalização, levando em consideração todos os seus elementos constitutivos e perpassando todo o espectro normal e anormal da externalização. As análises indicaram que um fator superordenado de externalização saturava a variância comum das 23 dimensões emocionais e comportamentais. Estas apresentam grande variedade de conteúdo, abrangendo todo um espectro de disposições relacionadas à alienação da culpa, impulsividade, empatia, agressividade e uso nocivo de substâncias. Esse estudo utilizou da versão brasileira do Inventário Espectral de Externalização, verificando a viabilidade clínica e psicométrica de duas versões reduzidas do instrumento (Nicácio, 2012).

Hopwood e Moser (2011) recuperaram uma estrutura de dois fatores simples utilizando o Personality Assessment Inventory, uma medida amplamente usada para avaliar traços da personalidade e psicopatologia em adultos. Seme- lhantemente a achados anteriores, a estrutura do modelo é invariante em relação ao sexo e à etnia.

Pesquisas sobre a estrutura fatorial de instrumentos de rastreamento psiquiátrico podem ser interpretadas como evidências de que os sintomas psicopatológicos são mais bem representados como dimensões de sofrimento subjetivo do que como categorias. O Questionário de Saúde Geral (QSG; de Carvalho et al., 2011) apresenta uma estrutura unifatorial que explica a covariância entre sinais e sintomas relacionados à depressão, somatização, ansiedade e à consolidação do sono, entre outros. Assim, pode-se afirmar que o QSG avalia uma dimensão que, em termos empíricos e conceituais, é semelhante ao construto internalização.

\section{Avaliação do Modelo: Comorbidade, Heterogeneidade Clínica e Utilidade Clínica}

A elaboração do Modelo Estrutural de Internalização e Externalização tem como base a construção de sistema de classificação que possibilite o entendimento de padrões de comorbidade diagnóstica e superar problemas relacionados à heterogeneidade clínica (Krueger \& Markon, 2006a). Esse modelo ainda teria importantes implicações conceituais no que tange a compreensão da relação entre psicopatologias e traços do temperamento e da personalidade (de Carvalho, 2012; Krueger et al., 2007) e a sobreposição neurobiológica e psicofarmacológica entre diferentes transtornos mentais (Patrick \& Bernat, 2006). Por fim, é um modelo apresentado como uma proposta para reformulação dos manuais oficiais de diagnóstico e classificação psiquiátrica, tendo um potencial efeito na conduta clínica de profissionais e pesquisadores em nível mundial (Goldberg, Krueger, Andrews, \& Hobbs, 2009; Krueger \& South, 2009).

A avaliação da pertinência desse modelo deve, portanto, observar se há evidências de que o modelo é capaz de superar as limitações as quais propõe superar. Ademais, devido às diversas implicações clínicas de uma taxonomia, é essencial avaliar o modelo do ponto de vista de sua utilidade clínica. 


\section{Comorbidade Diagnóstica e Sobreposição Neurobiológica}

Comorbidade foi um termo cunhado por Feinstein (1970) ao se referir sobre quaisquer condições clínicamente significativas que co-ocorrecem com a doença principal. $\mathrm{O}$ conceito de comorbidade, sob essa perspectiva, não apresenta repercução teórica, isto é: não há hipóteses sobre relação entre condições clínicas concomitantes, sua relevancia é devido a implicações por razões clínicas relaciondas ao tratamento e prognóstico. Entretanto, padrões de comorbidade entre transtornos mentais têm sido observados em diversos estudos clínicos e epidemiológicos, evidenciando que a co-ocorrência entre psicopatologias não se trata de um fenômeno casual que prescinda de uma abordagem explicativa.

A necessidade de entender o significado dos padrões de comorbidade motivou o exame da estrutura dos transtornos mentais que deflagrou duas dimensões latentes correlacionadas de internalização e externalização. Apesar de haver controvérsias quanto a viabilidade do modelo, internalização e externalização são dimensões que propõe explicar a co-ocorrência entre os transtornos mentais relacionados ao humor unipolar, à ansiedade, a conduta antissocial e ao uso noscivo de substâncias.

O mesmo raciocínio pretende explicar a sobreposição neurobiológica e o efeito de um mesmo fármaco em diferentes trantornos mentais: internalização e externalização explicam o que há de comum entre transtornos mentais, o que inclui o substrato neurobiológico comum entre eles (Patrick \& Bernat, 2006). Desse modo, a efetividade de fármacos específicos, como inibidores seletivos de recapitação de serotonina, sobre uma ampla gama de sinais, sintomas e transtornos é, provavelmente, devido ao seu efeito nessas dimensões gerais e não em elementos específicos de transtornos.

\section{Heterogeneidade Clínica}

Com base nos critérios diagnósticos do DSM-IV (APA, 2002), cinco sintomas em um conjunto de nove são suficientes para diagnosticar o transtorno depressivo maior. Esse modo de proceder gera diversas manifestações clínicas sob uma única rubrica diagnóstica. Tamanha heterogeneidade clínica produz muita inceteza quanto a validade das categorias nosológicas que conformam os sistemas oficiais de classificação dos transtornos mentais (Widiger \& Sankis, 2000). Por exemplo, não se sabe ao certo as diferentes manifestações do transtorno depressivo maior são subtipos do transtorno ou diferentes manifestações clínicas (van Loo, de Jonge, Romeijin, Kessler, \& Schoevers, 2012).

O Modelo Estrutural de Internalização e Externalização propõe que transtornos relacionados à depressão unipolar, ansiedade, uso de substâncias e conduta antisocial são indicadores unidimensionais das dimensões de internalização e externalização, mesmo quando avaliados no nível de sintomas (Wright et al., 2013). Neste modelo, a heterogeneidade clínica é conceitualmente acomodada pela perspectiva dimensional: níveis mais brandos de expressão dos traços (internalização e externalização) predisporiam poucos sintomas e de menor gravidade, enquanto manifestações mais intensas dos traços predisporiam sintomas mais diversificados e graves.

Entretanto, a fim de operacionalizar a avaliação dimensional de psicopatologias internalizantes e externalizantes, seria necessário a existência de um instrumento psicométrico padrão validado e normatizado. O Inventário Espectral de Externalização foi desenvolvido e validado, inclusive para o Brasil (de Carvalho et al., 2007), mas não há estudos de normatização. Não há instrumentos desenvolvidos que avaliem o espectro da internalização. O Personality Assessment Inventory (Hopwood \& Moser, 2011) não é uma medida clínica suficientemente ampla para ser usada como instrumento de psicodiagnóstico.

\section{Utilidade Clínica}

A utilidade clínica de um sistema diagnóstico pode ser definida por quatro principios fundamentais: facilidade de uso, conceituação de casos, fomento de uma comunicação clara (entre clínicos e clínico-paciente) e auxilio no planejamento do tratamento (First, 2005). Com exceção do primeiro quesito, todos os outros dependem diretamente do grau de validade e 
confiabiliade do modelo de classificação e diagnóstico em questão.

O conjunto de evidências favorávais ao Modelo Estrutural de Internalização e Externalização, conforme apresentado nesta revisão, indica elevado grau de validade e confiabilidade. Entretanto, para que o modelo se torne de fácil utilização é necessário o desenvolvimento de instrumentos de fácil aplicação clínica e com normas capazes de captar pequenas nuances e variações na população. O Inventário Espectral de Externalização, por exemplo, é excessivamente amplo (415 itens) e apesar de haver versões reduzidas validadas (Nicácio, 2012), não há normas disponíveis.

\section{Conclusão}

Tomadas as evidências em seu favor, o Modelo Estrutural de Internalização e Externalização apresenta bons indicadores de validade: os dados dão sustentabilidade aos pressupostos teóricos, há uma proposta sobre quais são e como funcionam os mecanismos psicobiológicos subjacentes aos construtos delineados e estes se mostram heurísticos. Por outro lado, sua pertinência como um modelo de classificação de psicopatologias apresenta virtudes e algumas limitações importantes. Dentre as primeiras, ressalta-se que o modelo é capaz de acomodar teórica e empiricamente os fenômenos de comorbidade diagnóstica e, parcialmente, heterogeneidade clínica. Todavia, não apresenta instrumental clínico para avaliação dos espectros da internalização e externalização, o que impossibilita o teste de sua implementação em contextos relevantes de aplicação clínica. Ademais, o modelo permanece aberto, uma vez que há dados inconclusivos quanto a estrutura fatorial da internalização e há estudos que sugerem que o modelo não representa um avanço empírico para os transtorno depressivos e de ansiedade em relação a estrutura oferecida pelos sistemas DSM-IV e CID-10.

\section{Referências}

American Psychiatric Association. (2002). Manual diagnóstico e estatístico de transtornos mentais:
$D S M-I V-T R$ (4. ed. rev., M. R. Jorge, Trad.). Porto Alegre, RS: ArtMed.

American Psychiatric Association. (2013). Diagnostic and statistical manual of mental disorders: $D S M-V\left(5^{\text {th }}\right.$ ed.) Arlington, VT: Author.

Clark, L. A. (2005). Temperament as a unifying basis for personality and psychopathology [Special section]. Journal of Abnormal Psychology, 114(4), 505-521. doi:10.1037/0021843X.114.4.505

Clark, L. A., Watson, D., \& Reynalds, S. (1995). Diagnostic and classification of psychopathology: Challenges to the current system and future directions. Annual Review of Psychology, 46, 121 153. doi:10.1146/annurev.ps.46.020195.001005

Cloninger, C. R., Svrakic, D. M., \& Przybeck, T. R. (1993). A psychobiological model of temperament and character. Archives of General Psychiatry, 50(12), 975-990. doi:10.1001/archpsyc.1993.01820240059008

De Carvalho, H. W. (2012). Da estrutura e dimensionalidade dos transtornos mentais comuns (Tese de doutorado, Universidade Federal de São Paulo, SP, Brasil).

De Carvalho, H. W., Andreoli, S. B., Lara, D. R., Patrick, C. J., Quintana, M. I., Bressan, R. A., ...Jorge, M. R. (2013). Structural validity and reliability of the Positive and Negative Affect Schedule (PANAS): Evidence from a large Brazilian community sample. Revista Brasileira de Psiquiatria, 35(2), 169-172. doi:10.1590/15164446-2012-0957

De Carvalho, H. W., Andreoli, S. B., Lara, D. R., Patrick, C. J., Quintana, M. I., Bressan, R. A., ... Jorge, M. R. (2014). The joint structure of major depression, anxiety disorders, and trait negative affect. Revista Brasileira de Psiquiatria. Advance online publication. doi:10.1590/15164446-2013-1329

De Carvalho, H. W., Andreoli, S. B., Vaidyanathan, U., Patrick, C. J., Quintana, I. M., \& Jorge, M. R. (2013). The structure of common mental disorders in incarcerated offenders. Comprehensive Psychiatry, 54(2), 111-116. doi:10.1016/j.comppsych.2012.07.

De Carvalho, H. W., Patrick, C. J., Jorge, M. R., \& Andreoli, S. B. (2011).Validation of the structural coherency of the General Health Questionnaire. Revista Brasileira de Psiquiatria, 33(1), 59-63. doi:10.1590/S1516-44462011000100012 
De Carvalho, H. W., Patrick, C. J., Krueger, R. F., Markon, K. E., \& Pinheiro, A. M. V. (2010). Validade de construto da versão Brasileira do Inventario Espectral de Externalização: Evidências a partir de uma amostra de estudantes universitários. Revista de Psiquiatria Clínica, 37(5), 206-211. doi:10.1590/S010160832010000500004

De Carvalho, H. W., Pinheiro, A. M. V., Patrick, C. J., Krueger, R. F., \& Markon, K. E. (2007). Tradução, adaptação cultural e análise de consistência interna do Inventario de Externalização. Avaliação Psicológica, 6(2), 217-227.

DeYoung, C. G., Peterson, J. B., Séguin, J. R., Mejia, J. M., Pihl, R. O., Beitchman, J. H., ...Palmour, R. M. (2006). The dopamine D4 receptor gene and moderation of the association between externalizing behavior and IQ. Archives of General Psychiatry. 63, 1410-1416. doi:10.1001/ archpsyc.63.12.1410

Feinstein, A. R. (1970). The pre-therapeutic classification of co-morbidity in chronic disease. Chronic Diseases, 23(7), 455-468. doi:10.1016/00219681(70)90054-8

First, M. B. (2005). Clinical utility: A prerequisite for the adoption of a dimensional approach in DSM. Journal of Abnormal Psychology, 114(4), 560564. doi:10.1037/0021-843X.114.4.560

Forbush, K. T., \& Watson, D. (2013). The structure of common and uncommon mental disorders. Psychological Medicine, 43(1), 97-108. doi:10.1017/S0033291712001092

Goldberg, D. P., Krueger, R. F., Andrews, G., \& Hobbs, M. J. (2009). Emotional disorders: Cluster 4 of the proposed meta-structure for DSM-V and ICD-11. Psychological Medicine, 39(12), 2043-2059. doi:10.1017/S0033291709990298

Hall, J. R., Bernat, E. M., \& Patrick, C. J. (2007). Externalizing psychopathology and the error-related negativity. Psychological Science, 18, 326333. doi:10.1111/j.1467-9280.2007.01899.x

Hettema, J. M., Neale, M. C., Myers, J. M., Prescott, C. A., \& Kendler, K. S. (2006). A populationbased twin study of the relationship between neuroticism and internalizing disorders. American Journal of Psychiatry, 163(5), 857-864. doi:10.1176/appi.ajp.163.5.857

Hopwood, C. J., \& Moser, J. S. (2011). Personality Assessment Inventory internalizing and externalizing structure in college students: In- variance across sex and ethnicity. Personality and Individual Differences, 50(1), 116-119. doi:10.1016/j.paid.2010.08.013

Insel, T. R., \& Quirion, R. (2005). Psychiatry as a clinical neuroscience discipline. JAMA, 294(17), 2221-2224. doi:10.1001/jama.294.17.2221

Kendler, K. S., Prescott, C. A., Myers, J., \& Neale, M. C. (2003). The structure and environmental risk factors for common psychiatric and substance use disorders in men and women. Archives of General Psychiatry, 60(9), 929-937. doi:10.1001/archpsyc.60.9.929

Kessler, R. C., Ormel, J., Petukhova, M., Mclaughlin, K. A., Green, J. G., Russo, L. J., ...Ustün, T. B. (2011). Development of lifetime comorbidity in the World Health Organization world mental health survey. Archives of General Psychiatry, 68(1), 90-100. doi:10.1001/archgenpsychiatry. 2010.180

Krueger, R. F. (1999). The structure of common mental disorders. Archives of General Psychiatry, 56(10), 921-926. doi:10.1001/archpsyc.56.10.921

Krueger, R. F., Caspi, A., Moffit, T. E., \& Silva, P. A. (1998). The structure and stability of common mental disorders (DSM-III-R): A longitudinalepidemiological study. Journal of Abnormal Psychology, 107(2), 216-227. doi:10.1037/0021843X.107.2.216

Krueger, R. F., Chentsova-Dutton, Y. E., Markon, K. E., Goldberg, D., \& Ormel, J. (2003). A crosscultural study of the structure of comorbidity among common psychopathological syndromes in the general health care setting. Journal of $A b$ normal Psychology, 112(3), 437-447.

Krueger, R. F., Hicks, B. M., Patrick, C. J., Carlson, S. R., Iacono, W. G., \& McGue, M. (2002). Etiologic connections among substance dependence, antisocial behavior, and personality: Modeling the externalizing spectrum. Journal of Abnormal Psychology, 111(3), 216-227.

Krueger, R. F., \& Markon, K. E. (2006a). Reinterpreting comorbidity: A model-based approach to understanding and classifying psychopathology. Annual Review of Clinical Psychology, 2006(2), 111-133.

Krueger, R. F., \& Markon, K. E. (2006b). Understanding psychopathology: Melding behavior genetics, personality, and quantitative psychology to develop an empirical based model. 
Current Directions in Psychological Science, 15, 113-117.

Krueger, R. F., Markon, K. E., Patrick, C. J., Benning, S. D., \& Kramer, M. D. (2007). Linking antisocial behavior, substance use, and personality: An intergrative quantitative model of the adult externalizing spectrum. Journal of Abnormal Psychology, 116(4), 645-666.

Krueger, R. F., Markon, K. E., Patrick, C. J., \& Iacono, W. G. (2005). Externalizing psychopathology in adulthood: A dimensional-spectrum conceptualization and its implications for DSM-V. Journal of Abnormal Psychology, 114(4), $537-$ 550. doi:10.1037/0021-843X.114.4.537

Krueger, R. F., \& South, S. C. (2009). Externalizing disorders: Cluster 5 of the proposed metastructure for DSM-V and ICD-11. Psychological Medicine, 39(12), 2061-2070. doi:10.1017/ S0033291709990328

Lara, D. R., Bisol, L. W., Brumstein, M. G., Reppold, C. T., de Carvalho, H. W., \& Ottoni, G. L. (2012). The Affective and Emotional Composite Temperament (AFECT) model and scale: A system-based integrative approach. Journal of Affective Disorders, 140(1), 14-37. doi:10.1016/j. jad.2011.08.036

Lara, D. R., \& Souza, D. O. (2001). Should we keep calling antidepressants antidepressants? Journal of Clinical Psychiatry, 62(10), 829-830.

Maj, M. (2005). Psychiatric comorbidity: An artefact of current diagnostic systems? The British Journal of Psychiatry, 182-184. doi:10.1192/ bjp.186.3.182

McGlinchey, J. B., \& Zimmerman, M. (2007). Examining a dimensional representation of depression and anxiety disorders' comorbidity in psychiatric outpatients with item response modeling. Journal of Abnormal Psychology, 116(3), 464474. doi:10.1037/0021-843X.116.3.464

Meehl, P. (1992). Factors and taxa, traits and types, differences of degree and differences in kind. Journal of Personality, 60(1), 117-174. doi:10.1111/j.1467-6494.1992.tb00269.x

Möller, H. J. (2008). Systematic of psychiatric disorders between categorical and dimensional approaches: Kraepelin's dichotomy and beyond. European Archives of Psychiatry and Clinical Neuroscience, 258(Suppl. 2), 48-73. doi:10.1007/s00406-008-2004-3
Nicácio, H. (2012). Avaliação psicométrica do Inventário de Externalização em uma amostra clínica (Dissertação de mestrado, Universidade Federal de Minas Gerais, Belo Horizonte, MG, Brasil).

Ofrat, S., \& Krueger, R. F. (2012). How research on the meta-structure of psychopathology aids in understanding biological correlates of mood and anxiety disorders. Biological Mood and Anxiety Disorders, 16(2), 13-22. doi:10.1186/20455380-2-13

Organização Mundial de Saúde. (1991). Compendio Internacional de Doenças (10. ed. rev.). Porto Alegre, RS: ArtMed.

Patrick, C. J. (2008). Psychophysiological correlates of aggression and violence: An integrative review. Philosophical Transactions of the Royal Society B: Biological Sciences, 12, 2543-2555.

Patrick, C. J., \& Bernat, E. M. (2006). The construct of emotion as a bridge to between personality and psychopathology. In R. F. Krueger \& J. L. Tackett (Eds.), Personality and psychopathology (pp. 174-209). New York: The Guilford Press.

Patrick, C. J., Bernat, E. M., Malone, S. M., Iacono, W. G., Krueger, R. F., \& McGue, M. (2006). P300 amplitude as an indicator of externalizing in adolescent males. Psychophysiology, 43(1), 84-92.

Reed, J. M., Roberts, M. C., Keeley, J., Catherine, H., Matsumoto, C., Sharan, P., ...Medina-Mora, M. E. (2013). Mental health professionals' natural taxonomies of mental disorders: Implications for the clinical utility of the ICD-11 and the DSM5. Journal of Clinical Psychology, 69(12), 11911212. doi:10.1002/jclp.22031

Røysamb, E., Kendler, K. S., Tambs, K., Ørstavik, R. E., Neale, M. C., Aggen, S. H., ...ReichbornKjennerud, T. (2011). The joint structure of DSM-IV Axis I and Axis II Disorders. Journal of Abnormal Psychology, 120(1), 198-209. doi: $10.1037 / \mathrm{a} 0021660$

Seeley, J. R., Kosty, D. B., Farmer, R. F., \& Lewinsohn, P. M. (2011). The modeling of internalizing disorders on the basis of patterns of lifetime comorbidity: Associations with psychosocial functioning and psychiatric disorders among first-degree relatives. Journal of Abnormal Psychology, 120(2), 308-321. doi:10.1037/ a0022621 
Slade, T., \& Watson, D. (2006). The structure of common DSM-IV and ICD-10 mental disorders in the Australian general population. Psychological Medicine, 36(11), 1593-1600. doi:10.1017/ S0033291706008452

Tellegen, A. (1982). Brief manual of the Multiphasic Personality Questionnaire. Unpublished manuscript, University of Minnesota, Minneapolis, MN, USA.

Vaidyanathan, U., Nelson, L. D., \& Patrick, C. J. (2012). Clarifying domains of internalizing psychopathology using neurophysiology. Psychological Medicine, 42(3), 447-459. doi:10.1017/ S0033291711001528

Van Loo, H. M., de Jonge, P., Romeijin, J. W., Kessler, R. C., \& Schoevers, R. (2012). Data-driven subtypes of major depressive disorder: A systematic review. BMC Medicine, 10, 156-168. doi:10.1186/1741-7015-10-156

Vollebergh, W., Iedema, J., Bijl, R., de Graaf, R., Smit, F., \& Ormel, J. (2001). The structure and stability of common mental disorders: The NEMESIS Study. Archives of General Psychiatry, 58(6), 597-603. doi:10.1001/archpsyc.58.6.597
Watson, D. (2005). Rethinking the mood and anxiety disorders: A quantitative hierarchical model of DSM-V. Journal of Abnormal Psychology, 114(4), 522-536. doi:10.1037/0021843X.114.4.522

Widiger, T. A., \& Clark, L. A. (2000). Towards DSM-V and the classification of psychopathology. Psychological Bulletin, 126, 946-962.

Widiger, T. A., \& Sankis, L. M. (2000). Adult psychopathology: Issues and controversies. Annual Review of Psychology, 51, 377-404. doi:10.1146/ annurev.psych.51.1.377

Wright, A. G., Krueger, R. F., Hobbs, M. J., Markon, K. E., Eaton, N. R., \& Slade, T. (2013). The structure of psychopathology: Toward an expanded quantitative empirical model. Journal of Abnormal Psychology, 122(1), 281-294. doi:10.1037/a0030133
Recebido: 04/11/2013

$1^{a}$ revisão: $24 / 02 / 2014$

Aceite final: $19 / 03 / 2014$

\section{Apêndice}

Tabela 1

Lista de Artigos Empíricos Identificados pela Busca Bibliográfica

\begin{tabular}{|c|c|c|c|c|c|c|c|}
\hline \multicolumn{8}{|c|}{ Artigos Empíricos } \\
\hline Autores & $N$ & Amostragem & País & Variáveis & Critério & Método & Classificação \\
\hline $\begin{array}{l}\text { Krueger et al., } \\
1998\end{array}$ & 1,037 & $\begin{array}{l}\text { Comunitária } \\
\text { Representativa } \\
\text { Probabilística }\end{array}$ & Austrália & $\begin{array}{l}\text { TD, TA, TC, } \\
\text { DA, DD }\end{array}$ & DSM-III-R & Longitudinal & $\begin{array}{c}\text { Epidemiológico- } \\
\text { estrutural }\end{array}$ \\
\hline Krueger, 1999 & 8,098 & $\begin{array}{l}\text { Comunitária, } \\
\text { Representativa, } \\
\text { Probabilística }\end{array}$ & EUA & $\begin{array}{l}\text { TD, TA, TC, } \\
\text { DA, DD }\end{array}$ & DSM-IV & Transversal & $\begin{array}{c}\text { Epidemiológico- } \\
\text { estrutural }\end{array}$ \\
\hline $\begin{array}{l}\text { Vollebergh et } \\
\text { al., } 2001\end{array}$ & 7,076 & $\begin{array}{l}\text { Comunitária, } \\
\text { Representativa, } \\
\text { Probabilística }\end{array}$ & Holanda & $\begin{array}{l}\text { TD, TA, TC, } \\
\text { DA, DD }\end{array}$ & DSM-IV & Longitudinal & $\begin{array}{c}\text { Epidemiológico- } \\
\text { estrutural }\end{array}$ \\
\hline $\begin{array}{l}\text { Krueger et al., } \\
2002\end{array}$ & 1,252 & Comunitária & EUA & $\begin{array}{c}\text { TC, TPA, } \\
\text { DA, DD, } \\
\text { Desinibição } \\
\text { (traço) }\end{array}$ & DSM-IV e MPI & Transversal & $\begin{array}{c}\text { Genética } \\
\text { comportamental }\end{array}$ \\
\hline $\begin{array}{l}\text { Kendler et al., } \\
2003\end{array}$ & 5,600 & Comunitária & EUA & $\begin{array}{l}\text { TD, TA, } \\
\text { TPA, DA, } \\
\text { DD }\end{array}$ & DSM-IV & Transversal & $\begin{array}{c}\text { Genética } \\
\text { comportamental }\end{array}$ \\
\hline $\begin{array}{l}\text { Krueger et al., } \\
2003\end{array}$ & 5,441 & Comunitária & 14 países & $\begin{array}{l}\text { TD, TA, DA, } \\
\text { ansiedade }\end{array}$ & $\begin{array}{c}\text { CID-10 e } \\
\text { Inventários } \\
\text { Psicométricos }\end{array}$ & Transversal & $\begin{array}{c}\text { Epidemiológico- } \\
\text { estrutural }\end{array}$ \\
\hline
\end{tabular}




\begin{tabular}{|c|c|c|c|c|c|c|c|}
\hline $\begin{array}{l}\text { Hettema et al., } \\
2006\end{array}$ & 8,000 & Comunitária & EUA & $\begin{array}{l}\text { TD, TA, } \\
\text { Neuroti- } \\
\text { cismo }\end{array}$ & $\begin{array}{c}\text { DSM-IV e } \\
\text { EFN-NEO-PI }\end{array}$ & Transversal & $\begin{array}{c}\text { Genética } \\
\text { comportamental }\end{array}$ \\
\hline $\begin{array}{c}\text { Krueger, } \\
\text { Markon, } \\
\text { Patrick, \& } \\
\text { Iacono, 2005 }\end{array}$ & 2,835 & Comunitária & EUA & $\begin{array}{l}\text { TPA, TC, } \\
\text { DA, DM, } \\
\text { DD }\end{array}$ & DSM-III-R & Transversal & Psicométrico \\
\hline $\begin{array}{c}\text { Slade \& } \\
\text { Watson, } 2006\end{array}$ & 10,641 & $\begin{array}{c}\text { Comunitária } \\
\text { Representativa, }\end{array}$ & Austrália & $\begin{array}{c}\text { TD, TA, } \\
\text { Neurastenia, } \\
\text { DA, DD }\end{array}$ & $\begin{array}{l}\text { DSM-IV e } \\
\text { CID-10 }\end{array}$ & Transversal & $\begin{array}{l}\text { Epidemiológico- } \\
\text { estrutural }\end{array}$ \\
\hline $\begin{array}{l}\text { Patrick et al., } \\
2006\end{array}$ & 969 & Comunitária & EUA & $\begin{array}{l}\text { TC, TPA, } \\
\text { DA, DD, } \\
\text { P300 }\end{array}$ & $\begin{array}{l}\text { DSM-III-R e } \\
\text { Eletroence- } \\
\text { falograma }\end{array}$ & Transversal & Psicofisiológico \\
\hline $\begin{array}{c}\text { Krueger \& } \\
\text { Markon, 2006a }\end{array}$ & 23,557 & $\begin{array}{c}\text { Comunitária } \\
\text { Representativa }\end{array}$ & $\begin{array}{c}\text { EUA, } \\
\text { Holanda e } \\
\text { Austrália }\end{array}$ & $\begin{array}{l}\text { TD, TA, } \\
\text { TPA, DA, } \\
\text { DD }\end{array}$ & DSM-IV & $\begin{array}{c}\text { Meta-análise } \\
\text { de estudos } \\
\text { transversais }\end{array}$ & $\begin{array}{l}\text { Epidemiológico- } \\
\text { estrutural }\end{array}$ \\
\hline $\begin{array}{l}\text { Hall et al., } \\
2007\end{array}$ & 92 & Universitários & EUA & $\begin{array}{l}\text { Espectro da } \\
\text { Externa } \\
\text { lização e } \\
\text { N100 }\end{array}$ & $\begin{array}{l}\text { Inventário } \\
\text { Espectral de } \\
\text { Externalização, } \\
\text { Eletroence- } \\
\text { falograma }\end{array}$ & Transversal & Psicofisiológico \\
\hline $\begin{array}{l}\text { Krueger et al., } \\
2007\end{array}$ & 1,787 & $\begin{array}{l}\text { Universitários } \\
\text { Prisioneiros }\end{array}$ & EUA & $\begin{array}{c}\text { Espectro da } \\
\text { Externa- } \\
\text { lização }\end{array}$ & $\begin{array}{c}\text { Inventário } \\
\text { Espectral de } \\
\text { Externalização }\end{array}$ & Transversal & Psicométrico \\
\hline $\begin{array}{c}\text { De Carvalho et } \\
\text { al., } 2007\end{array}$ & 258 & Universitários & Brasil & $\begin{array}{c}\text { Espectro da } \\
\text { Externa- } \\
\text { lização }\end{array}$ & $\begin{array}{c}\text { Inventário } \\
\text { Espectral de } \\
\text { Externalização }\end{array}$ & Transversal & Psicométrico \\
\hline $\begin{array}{l}\text { McGlinchey \& } \\
\text { Zimmerman, } \\
2007\end{array}$ & 2,300 & $\begin{array}{c}\text { Pacientes } \\
\text { Ambulatoriais }\end{array}$ & EUA & TD, TA & DSM-IV & Transversal & Psicométrico \\
\hline $\begin{array}{c}\text { De Carvalho, } \\
\text { Patrick, } \\
\text { Krueger, } \\
\text { Markon, \& } \\
\text { Pinheiro, 2010 }\end{array}$ & 258 & Universitários & Brasil & $\begin{array}{c}\text { Espectro da } \\
\text { Externa- } \\
\text { lização }\end{array}$ & $\begin{array}{c}\text { Inventário } \\
\text { Espectral de } \\
\text { Externalização }\end{array}$ & Transversal & Psicométrico \\
\hline $\begin{array}{l}\text { Kessler et al., } \\
2011\end{array}$ & 21,229 & $\begin{array}{l}\text { Comunitária } \\
\text { Representativa }\end{array}$ & 14 países & $\begin{array}{c}\text { TD, THb, } \\
\text { TA, } \\
\text { TImp, } \\
\text { TDPPI, DA, } \\
\text { DD }\end{array}$ & DSM-IV & Transversal & $\begin{array}{l}\text { Epidemiológico- } \\
\text { estrutural }\end{array}$ \\
\hline $\begin{array}{l}\text { Røysamb et } \\
\text { al., } 2011\end{array}$ & 2,794 & $\begin{array}{l}\text { Comunitária } \\
\text { Representativa } \\
\text { Probabilística }\end{array}$ & Noruega & $\begin{array}{c}\text { Eixo I e eixo } \\
\text { II }\end{array}$ & DSM-IV & Transversal & $\begin{array}{l}\text { Epidemiológico- } \\
\text { estrutural }\end{array}$ \\
\hline $\begin{array}{c}\text { Seeley et al., } \\
2011\end{array}$ & 816 & $\begin{array}{l}\text { Comunitária, } \\
\text { Representativa }\end{array}$ & EUA & $\begin{array}{c}\text { TD, TA, TA, } \\
\text { TC, DA, DD, } \\
\text { TP }\end{array}$ & DSM-IV & Transversal & $\begin{array}{l}\text { Epidemiológico- } \\
\text { estrutural }\end{array}$ \\
\hline $\begin{array}{c}\text { De Carvalho et } \\
\text { al., } 2011\end{array}$ & 146 & Comunitária & Brasil & $\begin{array}{c}\text { Sintomas } \\
\text { psicosso- } \\
\text { máticos, } \\
\text { depressivos, } \\
\text { de ansiedade, } \\
\text { ideação } \\
\text { suicida }\end{array}$ & $\begin{array}{l}\text { Questionário } \\
\text { de Saúde Geral } \\
\text {, }\end{array}$ & Transversal & Psicométrico \\
\hline
\end{tabular}




\begin{tabular}{|c|c|c|c|c|c|c|c|}
\hline $\begin{array}{l}\text { Hopwood \& } \\
\text { Moser, } 2011\end{array}$ & 246 & Universitários & EUA & $\begin{array}{l}\text { Sintomas } \\
\text { gerais e } \\
\text { traços }\end{array}$ & $\begin{array}{c}\text { Personality } \\
\text { Assessment } \\
\text { Inventory }\end{array}$ & Transversal & Psicométrico \\
\hline $\begin{array}{l}\text { De Carvalho, } \\
\text { Andreoli, } \\
\text { Vaidyanathan, } \\
\text { et al., } 2013\end{array}$ & 1,837 & $\begin{array}{l}\text { Prisioneiros } \\
\text { Representativa } \\
\text { Probabilística }\end{array}$ & Brasil & $\begin{array}{c}\mathrm{TD}, \mathrm{TA}, \mathrm{DA}, \\
\mathrm{DD}, \text { recidiva } \\
\text { criminal }\end{array}$ & DSM-V & Transversal & $\begin{array}{c}\text { Epidemiológico- } \\
\text { estrutural }\end{array}$ \\
\hline $\begin{array}{c}\text { Forbush \& } \\
\text { Watson, } 2013\end{array}$ & 16,233 & $\begin{array}{l}\text { Comunitária } \\
\text { Probabilística }\end{array}$ & EUA & $\begin{array}{c}\text { TD, THb, } \\
\text { TA, DA, DD } \\
\text { e TPA }\end{array}$ & DSM-IV & Transversal & $\begin{array}{l}\text { Epidemiológico- } \\
\text { estrutural }\end{array}$ \\
\hline $\begin{array}{l}\text { Wright et al., } \\
2013\end{array}$ & 8,841 & $\begin{array}{l}\text { Comunitária } \\
\text { Probabilística }\end{array}$ & Austrália & $\begin{array}{l}\text { Sintomas } \\
\text { diversos de } \\
\text { Psicopa- } \\
\text { tologia }\end{array}$ & DSM-IV & Transversal & $\begin{array}{c}\text { Epidemiológico- } \\
\text { estrutural }\end{array}$ \\
\hline
\end{tabular}

Notas. Legenda: TD, transtornos depressivos; TA, transtornos de ansiedade; TC, transtorno de conduta; DA, síndrome de dependência de álcool, DD síndrome de dependência de drogas; TPA, transtorno de personalidade antissocial; THb, transtornos de humor bipolar; TImp, Transtornos de controle de impulsos; TDPPI, transtornos diagnosticados pela primeira vez na infância ou adolescência; TA, transtornos da alimentação; DSM-III-R, Manual Diagnóstico e Estatístico dos Transtornos Mentais, $3^{\text {a }}$ Edição Revisada; DSM-IV, Manual Diagnóstico e Estatístico dos Transtornos Mentais, 4a Edição; CID-10, Classificação Internacional de Doenças, $10^{\mathrm{a}}$ edição.

Tabela 2

Artigos Teóricos e Críticos Identificados na Busca Bibliográfica

Ensaios Teóricos e Revisões de Literatura

Autores $\quad$ Assunto abordado

Widiger \& Sankis, 2000 Relativismo cultural sobre o diagnóstico psiquiátrico, excessiva comorbidade e heterogeneidade clínica.

Widiger \& Clark, 2000 Limitações das definições constitutivas e operacionais de "psicopatologia" e influência dos sistemas oficiais de classificação na produção acadêmica sobre o tema.

Maj, 2005 Como os sistemas oficiais de classificação e diagnóstico inflam artificialmente a comorbidade psiquiátrica.

Watson, 2005 Propõe uma reorganização dos transtornos de humor depressivo e de ansiedade com base em uma dimensão geral de internalização.

Clark, 2005 Mostra como os traços do temperamento são essenciais ao entendimento do significado das comorbidades psiquiátricas e relação entre personalidade e psicopatologia.

First, 2005 Discute a importância da utilidade clínica para uma nosologia psiquiátrica e as limitações no que tange a adoção de um modelo dimensional para a classificação de psicopatologias.

Krueger et al., 2005 Mostram que os transtornos externalizantes tem base etiológica comum e podem ser delimitados com base em um modelo de traço latente de um fator de ordem-superior, fator de Externalização.

Insel \& Quirion, 2005 Argumentam que os transtornos mentais devam ser tratados como "doenças do cérebro".

Krueger \& Markon, 2006b Propõe que a nosologia psiquiátrica adote uma postura empírica e teoricamente sustentada que permita mapear as comorbidade, agrupando as psicopatologias em termos de proximidade etiológica. 
Krueger \& Markon, 2006b Meta-análise que apresenta o Modelo Estrutural de Internalização e Externalização como um paradigma nosológico empírica e teoricamente orientado que supera as limitações da excessiva comorbidade psiquiátrica.

Möller, 2008 Avalia os princípios de sistematização da psicopatologia em termos de categorias ou dimensões.

Patrick, 2008 Revisa os correlatos neurofisiológicos da conduta antissocial e sua relação com o espectro da externalização.

Krueger \& South, 2009 Avaliam a validade do espectro da Externalização como base para a reorganização dos sistemas oficiais de classificação dos transtornos mentais.

Goldberg et al., 2009 Avaliam a validade do espectro da Internalização como base para a reorganização dos sistemas oficiais de classificação dos transtornos mentais.

Ofrat \& Krueger, 2012 Aborda a relação entre pesquisa sobre meta-estrutura em psicopatologia e os correlatos biológicos das psicopatologias.

Vaidyanathan et al., 2012 Avalia como marcadores psicofisiológico podem ajudar a caracterizar domínios de psicopatologias internalizantes. 